\title{
How many sentinel nodes should be harvested in oral squamous cell carcinoma?
}

\author{
Timo Atula • Taimur Shoaib • Gary L. Ross • \\ Henry W. Gray $\cdot$ David S. Soutar
}

Received: 26 March 2007 / Accepted: 19 November 2007 / Published online: 19 December 2007

(C) Springer-Verlag 2007

\begin{abstract}
The number of harvested lymph nodes when performing sentinel lymph node (SLN) biopsy remains controversial. The aim of this study was to examine the maximum number of nodes to be harvested for histopathological analysis. We also wanted to determine if the level of radioactivity within a SLN or its size were indicators for the likelihood of nodal metastases. The SLNs from 34 neck dissection specimens from patients with $\mathrm{T} 1 / \mathrm{T} 2 \mathrm{~N} 0$ oral and oropharyngeal carcinomas were included. Altogether 76 SLNs were measured for radioactivity and lymph node dimensions and volume. Tumour was identified in 16 of 76 nodes (positive nodes), and the remaining 60 nodes were free from tumour (negative nodes). In 9 of 16 cases, metastases were in the hottest node. Two patients had more than one positive SLN: the first and fourth hottest in one and the second and fourth hottest nodes in another contained tumour. However, all patients would have been staged accurately if only the hottest three sentinel nodes had been retrieved. Lymph nodes that contained tumour had a greater maximum diameter than non-metastatic SLNs. To stage the neck accurately, only the three hottest lymph nodes required sampling.
\end{abstract}

The corresponding author is a visiting Researh Fellow at the Canniesburn Unit. The contact e-mail address after August 2007 is timo.atula@hus.fi.

T. Atula $(\varangle) \cdot$ T. Shoaib · G. L. Ross · D. S. Soutar Canniesburn Plastic Surgery Unit, Jubilee Building, Royal Infirmary, 84 Castle Street, Glasgow G4 OSF, UK e-mail: timo.atula@northglasgow.scot.nhs.uk; timo.atula@hus.fi

H. W. Gray

Department of Nuclear Medicine,

Royal Infirmary, Glasgow G4 OSF, UK
Keywords Biopsy · Oral cavity · Oropharynx · Cancer . Diagnosis $\cdot$ Metastasis $\cdot$ Sentinel node biopsy $\cdot$ Neck

\section{Introduction}

Sentinel lymph node (SLN) biopsy has shown to be highly accurate in oral and oropharyngeal carcinomas [6, 10, 12]. One of the controversies surrounding SLN biopsy is how many lymph nodes should be harvested and whether all nodes identified as "sentinel" by dye and radiocolloid are true sentinel nodes. If several lymph nodes are required to be removed, the meaningfulness of SLN biopsy becomes questionable.

Since some of the colloid particles move from SLN to lymph nodes lower in the draining lymphatic basin, second and subsequent echelon nodes may sometimes be regarded as "sentinel nodes" [4]. It is not possible to determine by mere location whether a radioactive or blue node is a true first echelon lymph node. For example, a node highlighted by radiocolloid in level IV is as likely to be the first echelon node as is a lymph node in level II. Anatomically lower nodes are not necessarily second echelon nodes, unless efferent lymphatics, visualized, for example, by blue dye, can be seen draining from a node at a higher level into one at a lower level. It is a misconception that the first echelon SLN is the most radioactive, and more likely to contain a metastasis [13]. Moreover, SLNs which are largely infiltrated by the tumour are less radioactive [5]. Studies on melanoma show that little additional information is gained by following the radiotracer more distal to the primary site and removing more than two sentinel nodes [7].

Large lymph nodes are more likely to contain tumour than small lymph nodes, although it is known that a 
small lymph node may harbour a metastasis and a metastatic lymph node is not always the largest one [2, 14]. To our knowledge, the relationship between the SLN size and occurrence of nodal metastasis has not been reported previously in head and neck squamous cell carcinomas.

The primary aim of this study was to determine how many nodes should be harvested to accurately stage the neck. The secondary aim was to determine if the amount of radioactivity within a SLN or the relative size of a SLN was an indicator of the likelihood of nodal metastases.

\section{Patients and methods}

\section{Patients}

Thirty-one patients with previously untreated, clinically staged as T1/T2 N0, oral and oropharyngeal carcinomas were included. After harvesting the SLNs as described below, all patients underwent either selective neck dissection (levels I-III $n=2$; levels I-IV $n=12$ ) or modified radical neck dissection $(n=20)$. Altogether, 34 neck dissections were performed. Of the primary tumours 16 were in the tongue, seven in the floor of mouth, four in the retromolar trigone, three in the palate and one in the gingiva of the mandible.

Lymph nodes

All radioactive lymph nodes were harvested $20-24 \mathrm{~h}$ after the injection of up to $40 \mathrm{MBq}{ }^{99 \mathrm{~m}} \mathrm{Tc}$ labelled colloidal human serum albumin to the tumour site. After removal, radioactive SLNs were placed against the tip of the $14 \mathrm{~mm}$ collimated probe of the Neoprobe-1500 (Neoprobe Corp, Columbus, OH, USA) and a $10 \mathrm{~s}$ count was measured ex vivo.

Radioactive sentinel nodes were fixed in formalin and thereafter the three-dimensions (the maximum diameter, the middle diameter and the minimum diameter) of the node were measured. The volume of the lymph node was calculated using the formula: volume $\left(\right.$ in $\left.\mathrm{mm}^{3}\right)=4 / 3\left(\pi r_{1}\right.$ $r_{2} r_{3}$ ), where $r_{1}, r_{2}$ and $r_{3}$ were the radii of the three-dimensions. Lymph nodes were bisected, and if the two halves were thicker than $2 \mathrm{~mm}$, further sections were processed at $2 \mathrm{~mm}$ intervals. A single block from each section was examined using haematoxylin and eosin stain for the presence of tumour within the sentinel node.

Only radioactive nodes were included in this study; cold-blue SLNs (four in total, all non-metastatic) were not analysed because the intensity of the colour cannot be quantified in the same way as radioactivity. Altogether, 76 SLNs were harvested (range 1-6/neck side).

\section{Statistics}

For non-parametric tests, Mann-Whitney $U$ test and Pearson's correlation coefficient were used. For parametric tests, Student's $t$ test was used.

Ethics

The study was approved by the local research ethics committee and a written consent was obtained from all patients

\section{Results}

One or more hot (radioactive) SLNs were identified in 34 necks. Out of the 76 hot SLNs, 35 were hot only and 41 were hot and blue. Tumour was identified in 16 of 76 nodes (positive nodes), and the remaining 60 nodes were free from overt tumour (negative nodes). One hot node was found in 14 necks, two hot nodes in eight, three hot nodes in six, four hot nodes in three, five hot nodes in one and six hot nodes in two necks. Histopathological examination showed positive SLNs in 14 patients (14 necks), whereas 17 patients had negative SLNs only. Of the metastatic nodes, 11 were hot and blue whereas five were hot only.

\section{Radioactivity of SLNs}

In those patients with positive sentinel nodes, the hottest nodes were not always the nodes that contained tumour (Table 1). There were two patients with more than one positive sentinel node. In these two, the second and fourth hottest nodes and the first and fourth hottest nodes contained tumour. In the rest of the 16 sentinel nodes that contained tumour, no more than one node per patient contained metastases. In 9 of 16 cases, the hottest node contained metastases and in five cases, the hottest sentinel node did not contain tumour despite the presence of tumour in

Table 1 The rank (per patient) of radioactive nodes with their pathology

\begin{tabular}{llll}
\hline $\begin{array}{l}\text { Radioactivity of the } \\
\text { node in patients }\end{array}$ & \multicolumn{2}{l}{ Pathology of node } & Total \\
\cline { 2 - 3 } & Non-metastatic & Metastatic & \\
\hline Hottest node & 25 & 9 & 34 \\
Second hottest node & 15 & 4 & 19 \\
Third hottest node & 11 & 1 & 12 \\
Fourth hottest node & 4 & 2 & 6 \\
Fifth hottest node & 3 & & 3 \\
Sixth hottest node & 2 & & 2 \\
Total & 60 & 16 & 76 \\
\hline
\end{tabular}


another radioactive sentinel node. All patients would have been staged accurately if only the hottest three sentinel nodes had been retrieved.

The radioactivity of primary tumour sites between the groups of positive and negative nodes was comparable; there was no significant difference between the groups (Table 2). No correlation was detected between the radioactivity of the SLN and that of the tumour site (Pearson's correlation coefficient $(r)=0.08)$.

There was no difference in amount of radioactivity between positive and negative sentinel nodes $(p=0.45)$ (Table 2).

The dimensions of the SLNs

The maximum diameter of lymph nodes varied from 3 to $40 \mathrm{~mm}$ and the mean maximum diameter was $12.7 \mathrm{~mm}$ (95\% C.I.: $11.0-14.3 \mathrm{~mm}$ ) (Fig. 1). The volume of sentinel nodes varied from 3 to $6,409 \mathrm{~mm}^{3}$ and the mean volume was $457 \mathrm{~mm}^{3}$ (95\% C.I.: $249-664 \mathrm{~mm}^{3}$ ). The mean minimum and mean middle diameter of sentinel nodes was 5.2 and $7.4 \mathrm{~mm}$, respectively.

Lymph nodes that contained tumour had a greater maximum diameter than non-metastatic sentinel nodes (Figs. 1, 2, Table 2). The difference was highly significant $(p<0.001)$. A significant difference was also detected in the middle nodal dimension between positive and negative SLNs $(p<0.05)$. However, when comparing the minimum diameter or the volume of sentinel nodes for positive and negative nodes, the differences were not statistically significant. Even though the difference in the volume was large, it was not statistically significant ( $p=0.1$; Table 2 ).

The node with the largest maximum diameter was not always the sentinel node that contained metastases (Table 3). Although in 12 cases the node with the largest maximum diameter was the node containing metastases, in four cases, smaller nodes were the sentinel nodes which contained tumour. Two patients had more than one positive SLN in their neck: in one, the metastases were found in the largest and second largest nodes and in the other, the

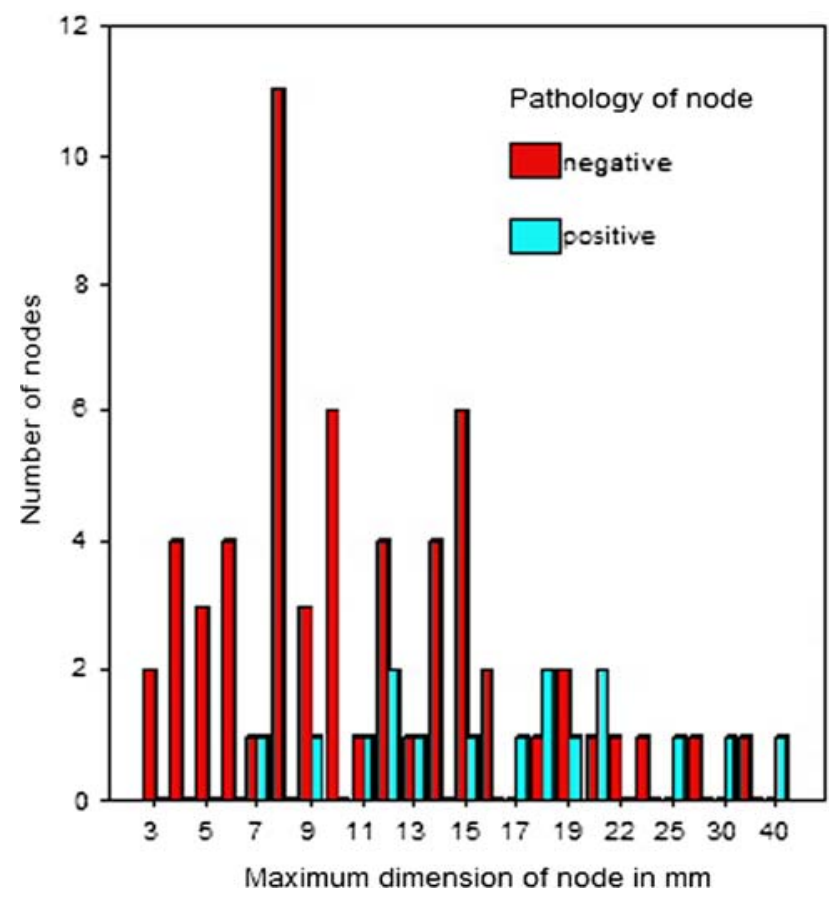

Fig. 1 Comparison of maximum nodal diameters of the metastatic (positive) and non-metastatic (negative) sentinel lymph nodes

Table 3 The rank of the maximum diametre of the sentinel node per case compared with the pathology of the node

\begin{tabular}{llll}
\hline \multirow{2}{*}{$\begin{array}{l}\text { Maximum diametre } \\
\text { of node per patient }\end{array}$} & \multicolumn{2}{l}{ Pathology of node } & \multicolumn{2}{c}{ Total } \\
\cline { 2 - 3 } & Non-metastatic & Metastatic & \\
\hline Largest node & 25 & 12 & 37 \\
Second largest node & 13 & 3 & 16 \\
Third largest node & 13 & 0 & 13 \\
Fourth largest node & 5 & 1 & 6 \\
Fifth largest node & 2 & & 2 \\
Sixth largest node & 2 & & 2 \\
Total & 60 & 16 & 76 \\
\hline
\end{tabular}

metastases were found in the largest and smallest (the fourth largest) nodes. Only the largest and second largest SLNs would have required sampling to stage the neck accurately.
Table 2 The characteristics related to the metastatic (positive) and non-metastatic (negative) SLNs

SLN sentinel lymph node

\begin{tabular}{llll}
\hline & Positive SLNs & Negative SLNs & $p$ value \\
\hline Number of SLN & 16 & 60 & \\
Tumour activity (mean; counts/10 s) & 18,900 & 18,042 & n.s. \\
Activity of SLN (mean; counts/10 s) & 980 & 1,554 & n.s. \\
Maximum diameter of SLN (mean in mm) & 18 & 11 & $p<0.001$ \\
Range (mm) & $7-40$ & $3-34$ & \\
$95 \%$ C.I. of mean (mm) & $13.4-22.3$ & $9.7-12.8$ & $p<0.05$ \\
Middle nodal dimension (mean in mm) & 9.5 & 6.8 & n.s. \\
Minimum diameter of SLN (mean in mm) & 6.3 & 4.9 & n.s. \\
Volume of SLN (mean in mm $^{3}$ ) & 789 & 368 & \\
\hline
\end{tabular}




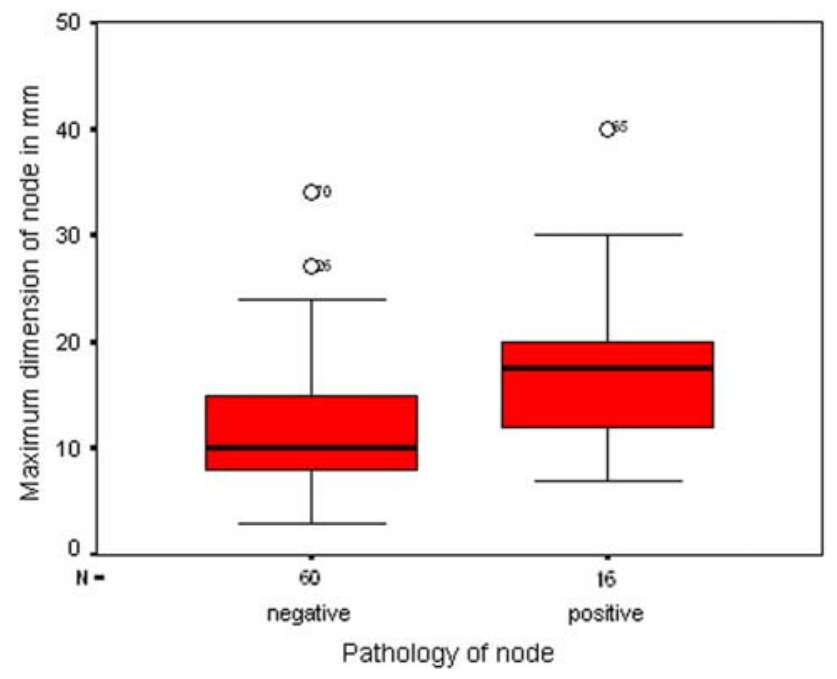

Fig. 2 Box plot comparing the maximum diameter of metastatic (positive) and non-metastatic (negative) sentinel lymph nodes, showing statistical significant differences

Radioactivity versus dimensions of the SLNs

A comparison of the radioactivity of the SLN and nodal volume or maximum nodal diameter showed almost no correlation (Pearson's correlation coefficient $(r)=0.008$ and $(r)=0.28$, respectively) (Fig. 3)

\section{Discussion}

This study was undertaken to determine whether nodal radioactivity levels and SLN size could be used to predict which nodes to sample in an oral cancer sentinel node

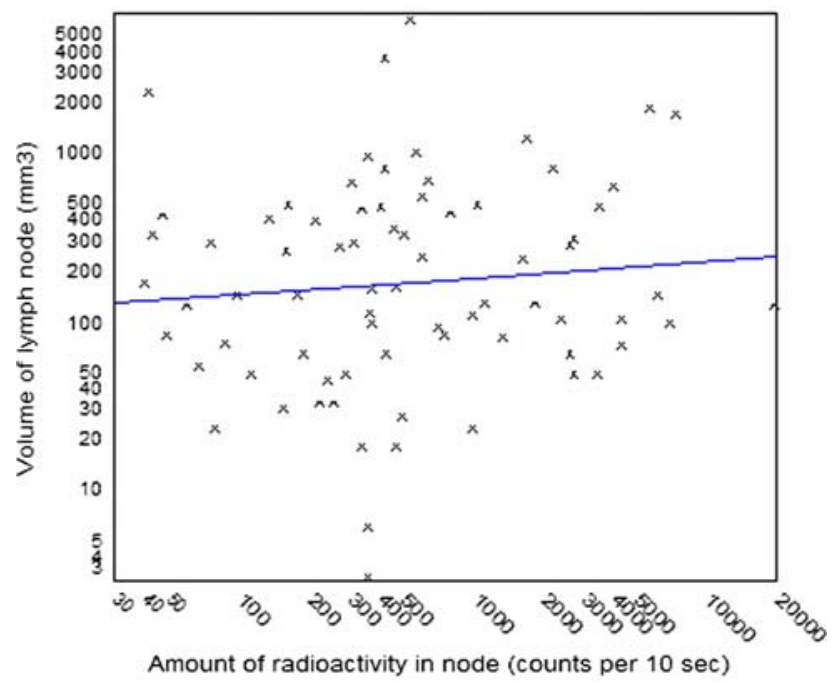

Fig. 3 A comparison of nodal volume and amount of radioactivity, showing the best fit curve. Pearson's correlation coefficient $(r)=0.008$ procedure. Within an individual case, the most radioactive SLNs with large maximum diameters were more likely to contain tumour than smaller, less radioactive nodes within the case.

The absolute amount of radioactivity within a sentinel node was no guide to the size of the node nor to the probability that the node contained tumour. However, within a single patient the converse was true-to accurately stage the neck, merely sampling the three hottest nodes would have been sufficient. Also, in each single case, a biopsy of the two largest SLNs would have been sufficient to accurately stage the neck, and the two largest nodes were always among the three hottest nodes.

More than one hot SLN was found in 20 cases. When the amount of radioactivity within SLNs was analysed in each patient, it was seen that the hottest nodes were more likely to contain tumour and the largest SLNs were most likely to be metastatic. Thus, in the 16 tumour containing SLNs, nine were the hottest nodes, four were the second hottest nodes and one was the third hottest node. The remaining two hot nodes were weakly radioactive, but were found in patients with more than one hot positive node. Our results are in line with those by Werner et al. [13], who reported that $39 \%$ of the cancer-positive necks would have been missed if only the hottest node had been excised.

Concerning the size, 12 of the 16 positive nodes were the largest sentinel nodes and three of the 16 were the second largest nodes. In the one remaining case, the fourth largest node contained tumour but it was also detected in a larger SLN. Our aim was not to find any specific size limit for metastasis, but to see the relative size of the SLNs that contained metastasis. It is known that very small lymph nodes can harbour a metastasis and a metastatic lymph node is not always the largest node in the neck dissection specimen [2, $3,14]$. It is also known that the nodal size of a SLN is an inaccurate predictor of nodal metastases [1]. Radiological criteria for metastatic lymph nodes have been assessed previously. "Central necrosis" caused by tumour involvement is a highly reliable sign of metastasis and can be detected also in small lymph nodes [2,3]. Different size criteria have been assessed in large series, but any size criteria, whether based on minimum or maximum dimensions or shape is unreliable when the lymph node is roughly normal size [2, $3]$. We made the measurements from the formalin fixed nodes. All nodes were, however, processed in a uniform manner and the relative size is therefore unlikely to be changed. Furthermore, in breast cancer tumour size can be measured either from fresh or fixed samples without affecting accuracy [8].

All necks were assessed clinically by palpation only. This may have led to the large diameters of some of the nodes we found in our results. Nevertheless, the accuracy in this series was $100 \%$. In addition, SLNs were not orien- 
tated, and so it is not possible to say whether these nodes would have been defined as malignant using radiological criteria.

The total volume of the lymph node was not significantly different between the two groups. This apparent paradox is probably due to the power of the study sample -16 sentinel nodes were found to contain tumour, in comparison to 60 , which were free from metastases.

Dynamic lymphoscintigraphy may sound like a suitable method to distinguish the first echelon nodes from other lymph nodes and thus be used as a guide for selecting the SLNs to be harvested. However, in the neck, it is not possible to distinguish whether a node appearing after the first one is really a second echelon node or if the later appearance is due to the longer lymphatic pathways. We also noticed that in cases of multiple SLNs all the nodes became visible quite simultaneously.

Although the actual amount of radioactivity within a sentinel node can only be estimated with a hand held gamma probe, such as the Neoprobe, it can be considered sufficient for the purposes of this study, since the hand held gamma probe is also used clinically to determine the radioactivity of the lymph nodes.

Blue dye was also used in search of SLNs in this series of patients, but we did not study its use because the intensity of the colour cannot be quantified in the same way as radioactivity or size. However, it is noteworthy that only a proportion of all SLNs as well as from metastatic nodes had been regarded as blue, and none of the cold blue nodes were metastatic. These findings support the conception that blue dye can be considered optional in search of SLNs.

In the present study, the histopathological sections were made by cutting the SLNs at $2 \mathrm{~mm}$ intervals only. Evidence is now emerging over how to process SLNs but at the time the study was performed all SLNs were processed in the same way as all nodes in the neck.

All patients in our series had a neck dissection. The sensitivity of the method at our unit has been reported previously, including the cohort within this patient population $[9,11]$. The aim of this study was not to focus on the nonsentinel lymph nodes, but it is noteworthy that in this patient series, consisting of $\mathrm{T} 1$ and $\mathrm{T} 2$ tumours, there were no false positive nodes, nor have been any instances of disease persistence outside of the dissected field during the follow-up of 5 years.

\section{Conclusion}

Our results indicate that for oral carcinomas it seems sufficient to harvest three of the hottest SLNs. Additionally, metastases are more likely to be in large SLNs and in all patients the necks would have been staged accurately as positive or negative if only the two largest SLNs had been retrieved. However, with the low power of this study, this conclusion should be interpreted with caution.

Conflict of interest statement The authors declare that they have no conflict of interest.

\section{References}

1. Alkureishi LW, Ross GL, MacDonald DG, Shoaib T, Gray H, Robertson G, Soutar DS (2007) Sentinel node in head and neck cancer: use of size criterion to upstage the no neck in head and neck squamous cell carcinoma. Head Neck 29:95-103

2. van den Brekel MW, Stel HV, Castelijns JA, Nauta JJ, van der Waal I, Valk J, Meyer CJ, Snow GB (1990) Cervical lymph node metastasis: assessment of radiologic criteria. Radiology 177:379_ 384

3. van den Brekel MW, Castelijns JA (1999) Radiologic evaluation of neck metastases: the otolaryngologist's perspective. Semin Ultrasound CT MR 20:162-174

4. Holmberg SB, Hafstrom L, Jacobsson L (1987) Phagocytosis and dynamic RES scintigraphy: an evaluation of commercial colloids in rats [published erratum appears in Nucl Med Commun 1987;8:1090]. Nucl Med Commun 8:335-346

5. Matsuzuka T, Kano M, Ohtani I, Miura T, Shishido F, Omori K (2005) Impact of sentinel node navigation technique for carcinoma of tongue with cervical node metastases. Auris Nasus Larynx 32:59-63

6. Paleri V, Rees G, Arullendran P, Shoaib T, Krishman S (2005) Sentinel node biopsy in squamous cell cancer of the oral cavity and oral pharynx: a diagnostic meta-analysis. Head Neck 27:739-747

7. Porter GA, Ross MI, Berman RS, Sumner WE 3rd, Lee JE, Mansfield PF, Gershenwald JE (2000) How many lymph nodes are enough during sentinel lymphadenectomy for primary melanoma? Surgery 128:306-311

8. Pritt B, Tessitore JJ, Weaver DL, Blaszyk H (2005) The effect of tissue fixation and processing on breast cancer size. Hum Pathol 36:756-760

9. Ross G, Shoaib T, Soutar DS, Camilleri IG, Gray HW, Bessent RG, Robertson AG, MacDonald DG (2002) The use of sentinel node biopsy to upstage the clinically N0 neck in head and neck cancer. Arch Otolaryngol Head Neck Surg 128:1287-1291

10. Ross GL, Soutar DS, MacDonald G, Shoaib T, Camilleri I, Roberton AG, Sorensen JA, Thomsen J, Grupe P, Alvarez J, Barbier L, Santamaria J, Poli T, Massarelli O, Sesenna E, Kovács AF, Grünwald F, Barzan L, Sulfaro S, Alberti F (2004) Sentinel node biopsy in head and neck cancer: preliminary results of a multicenter trial. Ann Surg Oncol 11:690-696

11. Shoaib T, Soutar DS, MacDonald DG, Camilleri IG, Dunaway DJ, Gray HW, McCurrach GM, Bessent RG MacLeod TI, Robertson AG (2001) The accuracy of head and neck carcinoma sentinel lymph node biopsy in the clinically N0 neck. Cancer 91:20772083

12. Stoeckli SJ, Pfaltz M, Ross GL, Stainert HC, MacDonald DG, Wittebkind C, Soutar DS (2005) The second international conference on sentinel node biopsy in mucosal head and neck cancer. Ann Surg Oncol 12:919-924

13. Werner JA, Dünne AA, Ramaswamy A, Dalchow C, Behr T, Moll R, Folz BJ, Davis RK (2004) The sentinel node concept in head and neck cancer: solution for the controversies in the N0 neck? Head Neck 26:603-611

14. Woolgar JA (1999) Pathology of the N0 neck. Br J Oral Maxillofac Surg 37:205-209 